\title{
Investigating the Affective Quality of Motion in User Interfaces to Improve User Experience
}

\author{
Doyun Park and Ji-Hyun Lee \\ Graduate School of Culture Technology, KAIST, 335 Gwahak-ro, \\ Yuseong-gu, Daejeon 305-701, Republic of Korea \\ \{soulvibe, jihyunlee\} @kaist.ac.kr
}

\begin{abstract}
This study focuses on motion in user interfaces as a design element which can contribute to an improved user experience of digital media entertainment. The design for user experience is necessary to deal with user emotion especially in the entertainment domain. As a means to approach emotion, we studied affective qualities which are the features of an artifact that can influence emotion. In user interface design, motion has not been practically dealt with in this perspective. Through empirical study, we verified that motion plays a significant role in forming the affective quality of user interfaces and found that content type and application type has influence on this effect. Moreover, a preliminary investigation was made on the use of the Effort system from Laban's theory for the design of motion in terms of affective quality.
\end{abstract}

Keywords: Motion, Affective quality, User interface, User experience.

\section{Introduction}

The emergence of user experience (UX) and diversifying entertainment content through digital media has brought new challenges to user interface design. Previously, the job of user interface designers was to ensure good usability so that users could operate the system at ease. But in the UX paradigm of today, it has become important to design for better experience by providing a holistic experience with consideration to emotion. This has greater significance in the entertainment domain than in most other areas because users fundamentally seek entertainment for an experience.

In order to approach emotion, designers need to consider the affective outcome of their design. According to Russell (2003) the features of an artifact that influence user's emotion is known as affective quality [1]. This refers to the feeling and impression that an artifact has which can cause changes in the user's emotion, such as the distinguishable feeling that Apple products convey. Therefore, for a better UX, the affective quality of every element in an artifact needs to be considered in design.

In this perspective, the focus of this study is on the design of motion in user interfaces. Motion has become an essential element of user interfaces through enhanced processor speed and bandwidth. Most interactive media, such as websites, IPTV, portable media players and mobile phones nowadays display many motions. However, unlike the static elements such as color and layout, the affective quality of motion has not received much attention. This is because motion was mainly used for instrumental purposes, such as giving feedback or attracting attention [2], [3]. 
With the growing use of motion, its relationship with the affective quality of user interfaces needs to be studied as a way to improve UX. Hence, the aim of this study is to first verify the influence which motion has on the affective quality of user interfaces through empirical investigation, and secondly investigate a method to design motion in user interfaces by applying a relevant framework.

\section{Related Work}

There have been many studies which dealt with the relationship between movement and emotion. Vaughan (1997) described the emotional effects that movement can have and the need to understand movement to enhance user's experience [4]. Bacigalupi (2000) also emphasized the need to handle movement with caution and suggested a conceptual framework for the design of movement in multimedia [5]. These studies provide the grounds for understanding movement, but the practical methods to actually design them for emotion are left to be studied in the context of user interfaces.

Studies on the methods to design movement for emotion have been conducted more frequently in the product design field. Lee (2007) studied the relationship between physical movement and emotion for application in interactive products, while Saerbeck (2010) looked into the emotional responses that robots can give with their movements [6], [7]. These studies reveal insights on how physical movement influence emotion. Nevertheless, the scope is often limited by examining the relationship of movement directly to emotion.

To gain a better understanding of how the movement element influences emotion, the relevant affective qualities need to be investigated as an intermediate step. This is because, as mentioned above, affective quality is one of the key factors which causes change in the user's emotion. Thus, to design elements in user interfaces towards an intended emotion, we need a practical and systematic method to control the elements in relation to affective quality.

\section{Theoretical Background}

\subsection{Framework of Movement}

The conceptual framework on movement by Bacigalupi (2000) describes two main qualities of movement [5]. One is the formal quality which includes rhythm, tempo, sequence, and direction. The other is the expressive quality which is composed of kinesis and empathy. The formal quality determines the form that the movements take while the expressive quality determines how each movement is expressed.

For our investigation on affective quality, we will focus on the expressive qualities of movement. The two elements of the expressive quality, kinesis and empathy, provide the frameworks which we can begin our study. Kinesis is about how a movement is expressed and it is described by the Effort system of Laban's theory. Empathy, on the other hand, is about the emotional engagement or physical identification related to a movement.

The relationship between kinesis and empathy provides a way to design for the affective quality of movement. By controlling kinesis, we can realize empathy accordingly. In this respect, the components of kinesis are reviewed in the next section for our study. 


\subsection{Laban's Theory of Effort}

Laban Movement Analysis (LMA) is a method developed by Rudolf Laban to interpret, describe, visualize and notate human movement. It is generally used to analyze the movement of dancers and athletes.

Laban's theories comprise of four main categories: Body, Effort, Shape, and Space $^{1}$. Out of these, Effort is a system used to understand the characteristics of the way a movement is performed with respect to inner intention. Thus it is closely related to the description of movement in terms of its quality and expressiveness. The Effort system is divided into four factors, each of which has two opposing elements as shown in table 1 [8].

Table 1. Description of the Effort system

\begin{tabular}{l|l|l}
\hline Effort Factor & Element & Description \\
\hline \multirow{2}{*}{ Space } & Indirect & flexible, meandering, wandering, multi-focus \\
\cline { 2 - 3 } & Direct & single focus, channeled, undeviating \\
\hline \multirow{2}{*}{ Weight } & Light & buoyant, easily overcoming gravity, decreasing pressure \\
\cline { 2 - 3 } & Strong & powerful, having an impact, increasing pressure \\
\hline \multirow{2}{*}{ Time } & Sustained & lingering, leisurely, indulging in time \\
\cline { 2 - 3 } & Sudden & hurried, urgent \\
\hline \multirow{2}{*}{ Flow } & Free & uncontrolled, abandoned \\
\cline { 2 - 3 } & Bound & controlled, restrained \\
\hline
\end{tabular}

Most movements show a combination of these factors. A combination which often occurs is of Space, Time and Weight. For example, a combination of Direct, Strong and Sudden elements would be embodied in a punching movement, while Indirect, Light and Sustained elements will result a floating movement. The three factors can combine to express eight different types of movement (Table 2) [9].

Table 2. Eight combinations of Space, Time and Weight factors

\begin{tabular}{l|ccc}
\hline Movement type & Space & Time & Weight \\
\hline Slashing & Indirect & Sudden & Strong \\
Gliding & Direct & Sustained & Light \\
Pressing & Direct & Sustained & Strong \\
Flicking & Indirect & Sudden & Light \\
Wringing & Indirect & Sustained & Strong \\
Dabbing & Direct & Sudden & Light \\
Punching & Direct & Sudden & Strong \\
Floating & Indirect & Sustained & Light \\
\hline
\end{tabular}

${ }^{1}$ The terms referring to the theories of LMA are capitalized to distinguish them from their commonly used meanings. 
Although the Effort system was devised for the study of human body movement, it provides a systematic way to describe the expressive quality of movement with the four factors. Thus, it is an effective and practical framework to work with. In our study, we will investigate the use of the Effort system for the design of motion in user interfaces.

\section{User Study on Motion and Affective Quality}

A user study was performed to verify the significance of the motion element on the affective quality of user interfaces. This user study aims to investigate the following points:

- The influence of motion on the affective quality of user interfaces.

- The dependency of this influence on two contextual factors: content type (e.g. the genre of the content) and application type (e.g. interface for music, movies, photos, etc.).

\subsection{Prototypes}

Three different interfaces were developed for the user study by Adobe Flash CS3: a minimal interface showing a moving object (Fig. 1) and two image viewing interfaces with different contents - one with soccer match images and the other with tropical resort images (Fig. 2). The minimal interface and the image viewing interfaces were developed to investigate the influence of application type. As for the image viewing interfaces, two distinctly different contents were implemented to investigate the influence of content type.

Three types of motion were applied to these prototypes. Two of these motions were designed to display opposing combinations of the Space, Time, and Weight factors of the Effort system. With the parameters adopted from Chi (1999), motion A had parameter settings for Direct, Sudden, and Strong elements while motion B had

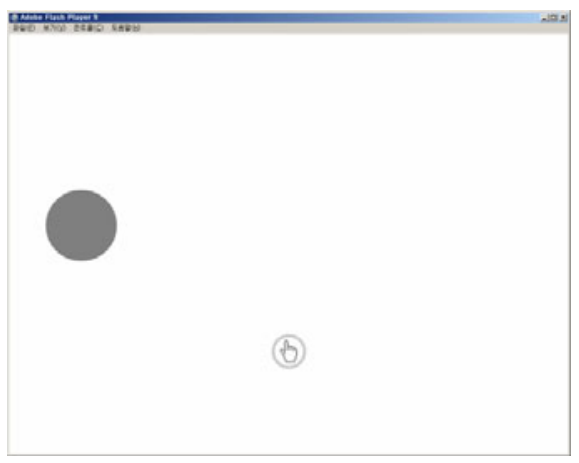

Fig. 1. The minimal interface displays an object that shows motion at user input. It consists of minimal elements to make the users focus on motion. It. Different types of motion are shown as the object changes position on the $\mathrm{x}$-axis. 

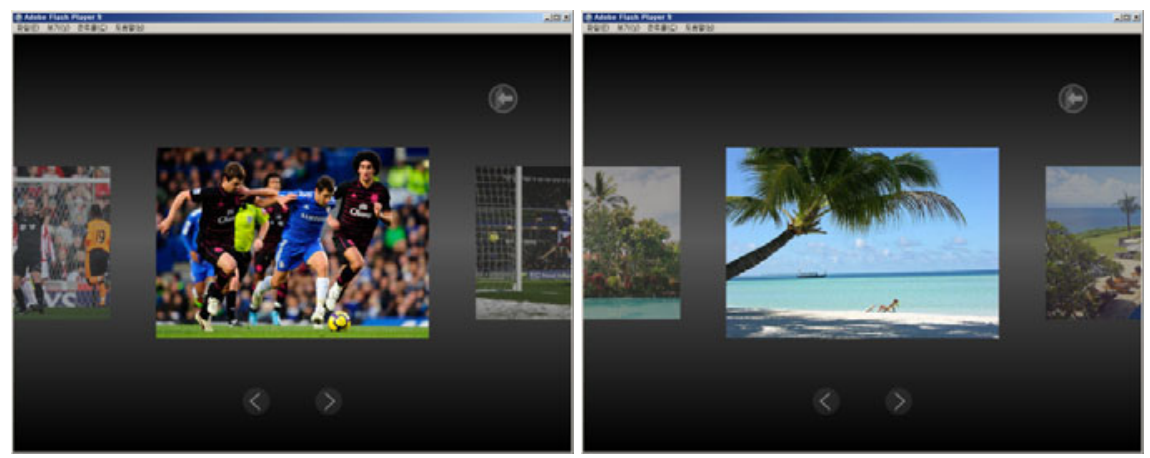

Fig. 2. The image viewing interface allows users to browse through a set of photos as they shift horizontally from one to another on input. Two types of content were implemented in this interface: one that shows soccer match images (left) and the other that shows tropical resort images (right).

Table 3. Motion parameters applied to prototypes

\begin{tabular}{l|ccc}
\hline Property & Motion A & Motion B & Motion C \\
\hline Path curvature & Straight & Curved & $\mathrm{n} / \mathrm{a}$ \\
Duration & $0.4 \mathrm{sec}$ & $3.6 \mathrm{sec}$ & $0 \mathrm{sec}$ \\
Acceleration & Acceleration & Deceleration & $\mathrm{n} / \mathrm{a}$ \\
Anti./Oversh. & Anticipation & Overshoot & $\mathrm{n} / \mathrm{a}$ \\
\hline
\end{tabular}

parameter settings for Indirect, Sustained, and Light elements (Table 3) [8]. Only the four properties which were generally applicable on an abstract object were applied: the path curvature property determines the straightness or roundness of the path that the object moves through, the duration property determines the length of time the motion occurs, the acceleration property determines the rate of change in velocity, and the anticipation/overshoot property determines if there is a slight pull before the motion begins or if there is a slight swing out at the end of the motion.

Motion $\mathrm{C}$ is a common type where an object changes position instantly without any motion in-between. This motion was implemented to see how much more influence dynamic motions can have over the common minimal use of motion. All prototype interfaces were presented with Adobe Flash Player on a 20 inch touch screen monitor. User input was made by tapping the objects on the screen.

\subsection{Experiment Design}

In our experiment, motion was the first independent factor and context was the second independent factor. To investigate the influence of these two factors, the perceived affective qualities were measured for the three types of user interfaces, each of which displayed the three types of motions mentioned above. A within-subject design was used to reduce the possible influence of individual differences.

For measurement, the following seven types of affective qualities were surveyed on a five point Likert scale: refreshing, calm, luxurious, powerful, youthful, uniqueness and 
futuristic. These affective qualities were adopted from Hong (2009) where the seven types were classified as the prevailing affective qualities in website interfaces [10].

\subsection{Procedure}

20 graduate students, 12 male and 8 female, aged from 23 to 29, participated in the experiment. First, the participants rated the affective quality of the three motion types displayed by the minimal interface. Next, they experienced and rated the image viewing interfaces: three sessions for each motion types on both the soccer match images and tropical resort images.

For the image viewing interface, different image sets were presented in every session to prevent bias from seeing same images repeatedly. At the same time, to cancel out the influence of the differing images on the evaluation of affective quality, the images within each content type were selected from identical sources and the image sets were paired randomly with motion types for every participant.

\subsection{Result}

The ratings on the affective qualities varied according to motion type in all three interfaces. Multivariate analysis of variance (MANOVA) was used to analyze the influence of the two independent factors on the set of affective qualities. We could verify that the motion factor had a significant influence on all tested affective qualities in all three interfaces at the $p<.05$ level. On the other hand, the contextual factors (content type and application type) only showed significant influence on some affective qualities for some motions.

In the soccer match viewing interface, motion B significantly lowered the refreshing, powerful, and youthful affective quality than the other motions while significantly increasing the calm affective quality of the interface (Fig. 3). On the other hand,

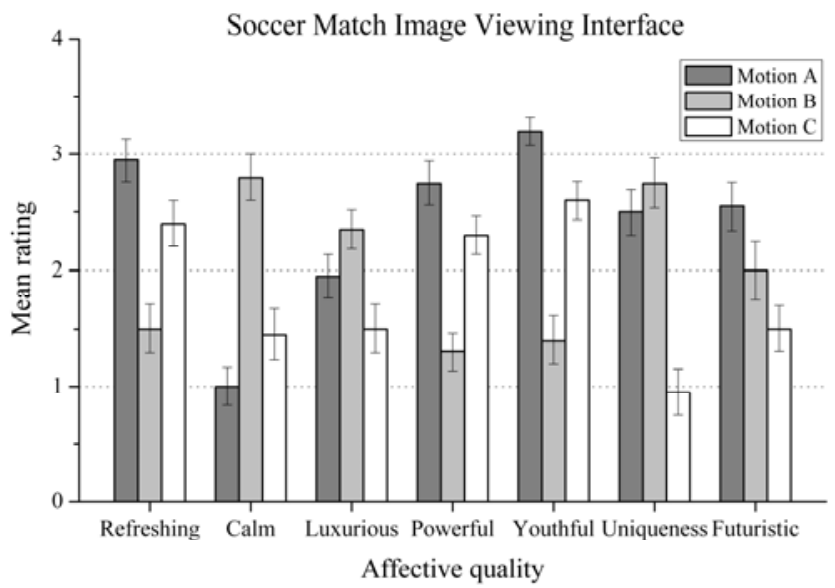

Fig. 3. Mean ratings on the affective qualities of the soccer match image viewing interface show statistically significant differences according to motion type. Error bars represent the standard error of the mean. 


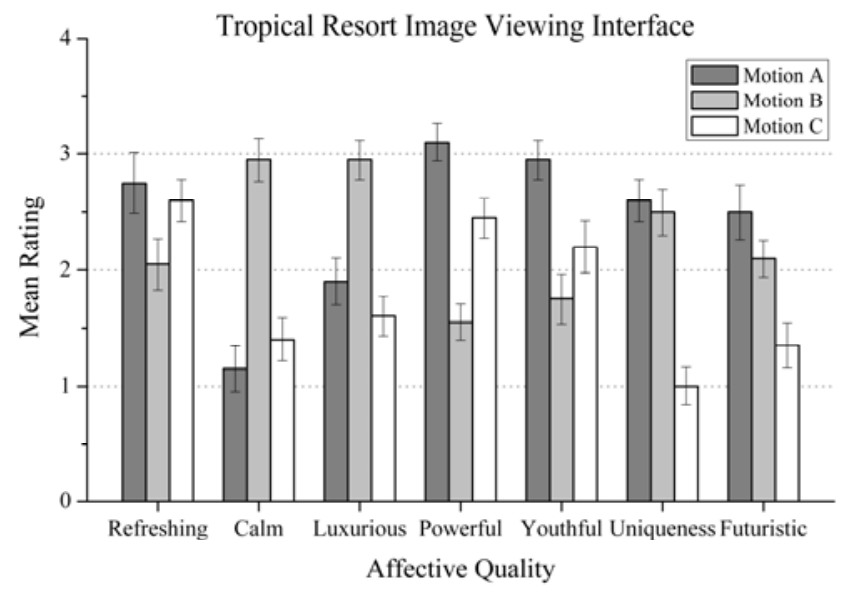

Fig. 4. Mean ratings on the affective qualities of the tropical resort image viewing interface show statistically significant differences according to motion type.

motion A significantly increased the youthful affective quality while motion $\mathrm{C}$ lowered the uniqueness affective quality in comparison to other motions. Similar influence was found from the tropical resort content (Fig. 4).

Content type showed little significance on how motion influences the affective quality of user interfaces. The ratings of each motion were compared among the soccer match and tropical resort image viewing interfaces. Only motion B showed a significantly differing level of influence on the luxurious affective quality by strengthening it in more the tropical resort content than in the soccer match content. Nevertheless, the rank order of motion type on the level of influence for each affective quality was generally identical among the two content types.

Next, the affective qualities measured from the minimal interface were examined. The three motions used in this experiment showed statistically significant influence on all affective qualities at the $p<.05$ level except uniqueness. In particular, motion B in this interface significantly lowered the refreshing, powerful and youthful affective qualities and significantly increased the calm and luxurious affective qualities than other motions (Fig. 5).

As a result, application type had greater significance on the influence of motion than content type. Rating comparison between the image viewing interfaces and the minimal interface showed significant difference in how much motion influenced the calm, luxurious, powerful, uniqueness, and futuristic affective qualities. Moreover, the rank order of motion type on the level of influence was not identical to the image viewing interfaces for the uniqueness and futuristic affective qualities.

\subsection{Discussion}

From the above results and statistical analyses, we were able to verify that the motion element has a significant influence on the seven affective qualities of user interface that were tested. 


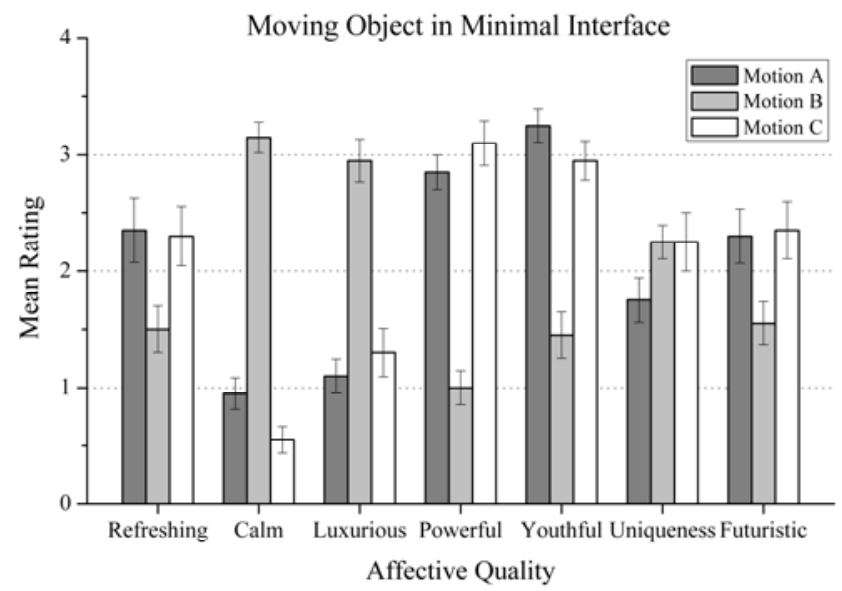

Fig. 5. Mean ratings on the affective qualities of the moving object show statistically significant differences by motion type except for the uniqueness affective quality.

Content type showed partial influence on how motion affects affective quality. Motion B had greater influence on the luxurious affective quality in the tropical resort content than in the soccer match content. This difference is analyzed to be the result of the users' attitude being more accepting to motion B within the tropical resort content than in the soccer match content because its leisurely feel is expected from the relaxing resort images. From this analysis, we can understand how content type acts as a variable. However, this influence is not so profound as to change one motion to have a dominant effect in one content type and not in another.

Lastly, the influence of application type was examined by comparing the results from the minimal interface and the image viewing interfaces. The overall result from the moving objects was similar to the image viewing interfaces. However, the influence of motion $\mathrm{C}$ on the uniqueness and futuristic affective quality was significantly greater in the moving object interface. This difference is analyzed to result from the user's differing expectations on each application types. The uniqueness and futuristic feel by motion $\mathrm{C}$ was not so significant in image viewing interfaces because users were accustomed to photo images shifting instantly in websites.

Our analysis shows that motion should be considered as a factor of affective quality in user interfaces. Moreover, the content type and application type requires examination in the user's perspective to accurately predict the effect of motion.

\section{User Study on the Properties Related to the Effort System}

The verified significance of motion provides motivation for a practical method to design motion. To do this, we began an investigation on the use of the Effort system of Laban's theory because, by being able to control motion in terms of this framework, the affective qualities of motion can be controlled and communicated more effectively. As a preliminary stage of this investigation, we performed a user study to identify the properties in a user interface that relate to three factors of the Effort system in comparison to human body movement. 


\subsection{Prototype}

A minimal interface that displays eight different types of motion through a moving object was developed using Adobe Flash CS3. Each motion was designed to be a combination of the Space, Time and Weight factors of the Effort system (punching, floating, gliding, wringing, pressing, dabbing, slashing and flicking).

The parameters for each combination were adopted from the observations made on human body movements in [8]. The parameters applied were related to four motion properties which are generally applicable on an abstract object in user interfaces: path curvature, duration, acceleration, and anticipation/overshoot (Table 4). The applied level of the acceleration and anticipation properties varied for some Effort combinations according to the observations in [8]. All motions were presented with Adobe Flash Player on a 20 inch touch screen monitor. The object displayed motion when the participant tapped the object.

Table 4. Motion parameters applied for each element of the three Effort factors

\begin{tabular}{l|c|c|c|c|c|c}
\hline \multirow{2}{*}{ Property } & \multicolumn{2}{|c|}{ Space } & \multicolumn{2}{c|}{ Time } & \multicolumn{2}{c}{ Weight } \\
\cline { 2 - 7 } & Indirect & Direct & Sustained & Sudden & Light & Strong \\
\hline Path Curvature & curved & straight & - & - & - & - \\
Duration & - & - & long & short & - & - \\
Acceleration & - & - & decelerate & accelerate & decelerate & accelerate \\
Anti./Oversh. & - & - & overshoot & none & overshoot & anticipation \\
\hline
\end{tabular}

\subsection{Experiment Design}

We tested four motion properties which were the independent factors and measured how the three Effort factors were perceived. For measurement, five point semantic differential scales were used on each Effort factors: the Space factor ranging from Indirect to Direct, the Time factor ranging from Sustained to Sudden, and the Weight factor ranging from Light to Strong.

\subsection{Procedure}

21 graduate students, 10 male and 11 female, aged from 23 to 32, participated in the experiment. Eight motions representing the Effort combinations were shown to participants in random order. After sufficiently viewing the motion, participants rated how it was perceived in terms of the three Effort factors.

\subsection{Result}

For all the motions tested, the Space and Time factors were perceived as expected by the parameters applied. With the Weight factor however, the parameters applied for dabbing, flicking, pressing and wringing motions were perceived opposite to what was expected. The parameters applied for dabbing and flicking were perceived as Strong while the parameters applied for pressing and wringing were perceived as Light. 
MANOVA was performed to identify the effect of the four properties that were applied. Path curvature had a significant influence on the Space factor while duration and acceleration had significant influences on the Time and Weight factors at the $p<.05$ level. On the other hand, anticipation/overshoot did not have significant influence on any of the three Effort factors (Fig. 6).
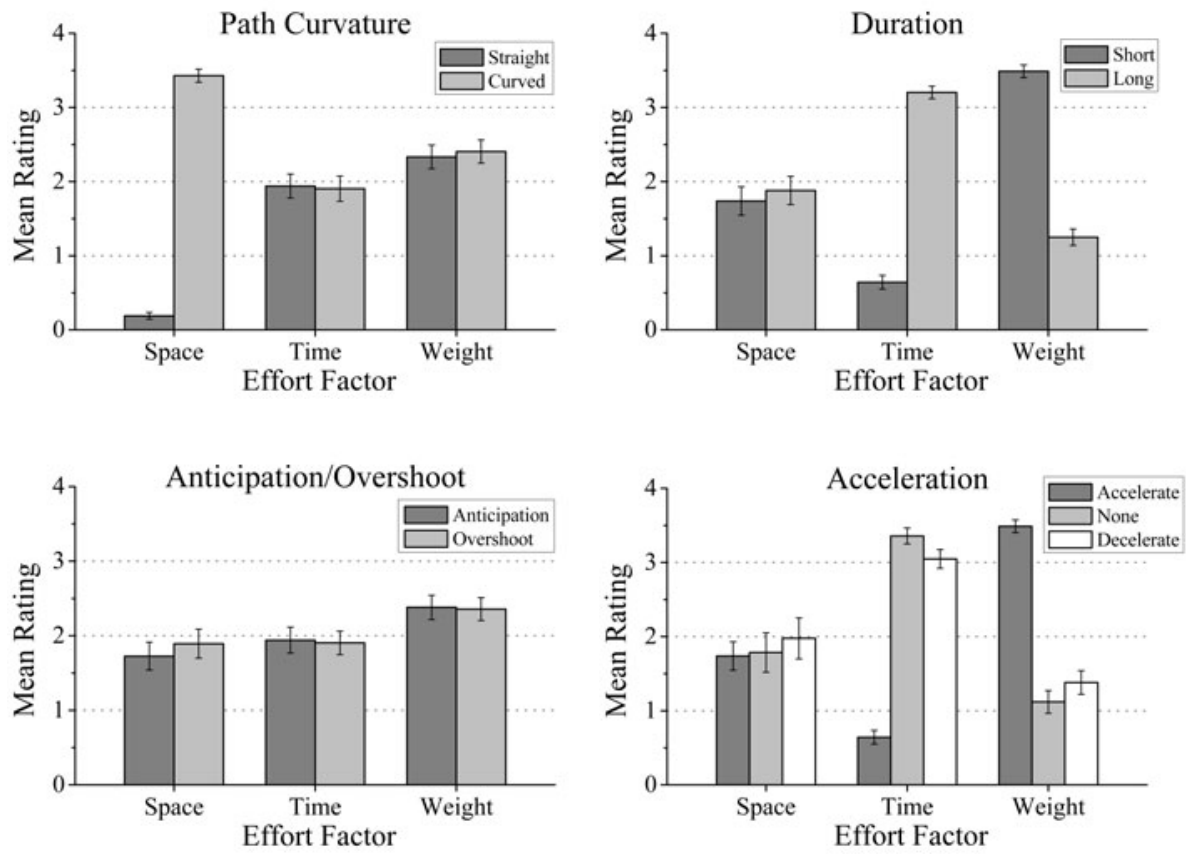

Fig. 6. Mean ratings of each Effort factors relative to the parameters of the four properties. Ratings close to 0 were perceived to be Direct, Sudden and Light, while values close to 4 were perceived to be Indirect, Sustained and Strong for each of the Space, Time and Weight factors, respectively. The error bars represent the standard error of the mean.

\subsection{Discussion}

From this user study, we were able to identify the motion properties that can be used to control motion in terms of the three Effort factors in user interfaces. In coherence with human body movement, path curvature was related to the Space factor, duration was related to the Time factor, and acceleration was related to both Time and Weight factor in user interfaces.

Differences were also discovered. In human body movements, the anticipation/overshoot property was observed to be related to the Time and Weight factor. However, it did not show relation to any factors in the user interface setting. On the other hand, the duration property showed strong relation to the Weight factor in user interfaces while this relation was not observed in human body movement. This 
explains why four of the motions types tested were perceived as having the opposite element on the Weight factor.

Unlike human body movements, we can assume that duration and acceleration plays a significant role in the perception of the Weight factor in user interfaces. These findings imply that the properties of motion act differently on the perception of affective quality in user interfaces when compared to human body movements.

\section{Conclusion and Future Work}

Through empirical study, we confirmed that motion significantly contributes to the affective quality of user interfaces. The level of influence by motion varied according to the content type and application type for several affective qualities. This effect is likely to have come from varying user expectations according to the two contextual factors. Thus, user expectation must be studied when designing motions for the affective quality of user interfaces. Overall, our result provides a motivation towards the study of motion design for affective quality.

Our second study identified the motion properties related to the three Effort factors in user interfaces and compared it with the relations found in human body movements. The investigated properties showed several differences in how they influence the Effort factors in user interfaces. These results imply that it is inadequate to directly adopt the human body movement parameters of the Effort system for motions in user interfaces. Further investigation on the properties is required to feasibly apply the Effort system for the design of motion in user interfaces.

For future work, an in-depth study on how the motion properties relate to the Effort factors is required. Moreover, studies on a wider range of users are required for practicality, since our experiments were performed on a limited number and cultural range of participants. Also, an investigation on the formal qualities of motion would be necessary to expand the variety of affective qualities designable in user interfaces. This knowledge will provide leverage in designing motions for satisfying affective qualities in user interfaces which can improve the UX of entertainment in digital media.

\section{References}

1. Russell, J.A.: Core Affect and the Psychological Construction of Emotion. Psychological Review 110, 145-172 (2003)

2. Stone, D., Jarrett, C., Woodroffe, M., Minocha, S.: User Interface Design and Evaluation. Morgan Kaufmann, Amsterdam (2005)

3. Galitz, W.: The Essential Guide to User Interface Design. Wiley, New York (2002)

4. Vaughan, L.C.: Understanding Movement. In: SIGCHI conference on Human factors in computing systems 1997, pp. 548-549 (1997)

5. Bacigalupi, M.: Designing Movement in Interactive Multimedia. Interfaces 44, 12-15 (2000)

6. Lee, J.-H., Park, J.-Y., Nam, T.-J.: Emotional Interaction through Physical Movement. In: Jacko, J.A. (ed.) HCI 2007. LNCS, vol. 4552, pp. 401-410. Springer, Heidelberg (2007) 
7. Saerbeck, M., Bartneck, C.: Perception of Affect Elicited by Robot Motion. In: 5th ACM/IEEE international conference on Human-robot interaction, pp. 53-60 (2010)

8. Chi, D.M.: A Motion Control Scheme for Animating Expressive Arm Movements. Ph.D. Dissertation, University of Pennsylvania (1999)

9. Laban, R., Lawrence, F.C.: Effort: Economy in Body Movement. Plays, Inc., Boston (1974)

10. Hong, S.-Y., Lee, H.-J., Jin, K.-N.: A Study on the Image Scale through the Classification of Emotion in Web Site. Science of Emotion \& Sensibility 12, 1-10 (2009) 